Stroke

\section{Stroke in patients with human immunodeficiency virus infection}

\section{Myles Connor}

\section{What is the impact of HIV and HIV therapy on the nature of stroke and stroke management?}

S troke occurring in the human immunodeficiency virus (HIV) infected patient is a frequent clinical challenge for clinicians working in regions with a high prevalence of HIV. Is the stroke a co-occurrence of two common conditions, or is it the direct or indirect result of the HIV infection and, if so, how? HIV infection causes stroke by predisposing to opportunistic infections such as tuberculosis and fungal meningitis, by increasing cardioembolic stroke due to direct cardiac involvement with HIV, possibly by interfering with blood coagulation through antiphospholipid antibodies or reduced protein $\mathrm{S}$, or by causing an arteriopathy. In the paper by Tipping and colleagues ${ }^{1}$ in this issue of $J$ Neurol Neurosurg Psychiatry, the authors describe their experience of HIV associated stroke in a large prospective stroke register from Cape Town, South Africa (see page 1320).

Sixty-seven $(6 \%)$ of 1087 stroke patients were HIV positive. The majority of the HIV infected patients were younger than 46 years and had suffered ischaemic strokes. Most ischaemic strokes were caused by infectious meningitides, coagulopathies and vasculopathy. They diagnosed HIV associated vasculopathy in patients with extracranial carotid occlusion or near occlusion thought not to be due to atherosclerosis or any other identifiable cause, or with intracranial vasculopathy in medium vessels diagnosed radiologically on the basis of vessel occlusion, stenosis or vessel calibre variation without another cause. Histology in one case showed leucocytoclastic vasculitis of the vaso vasorum in the extracranial arteriopathy without evidence of HIV antigen, and the authors have previously described intimal changes in the intracranial medium arteriopathy of another. ${ }^{2}$ Patients with extracranial vasculopathy had higher CD4 counts than those with intracranial vasculopathy.

This study adds to the sparse literature on stroke in HIV infected patients, particularly from Africa. However, not all patients were systematically tested for HIV and it is not clear whether older patients were as likely as younger patients to be tested for HIV, or if other factors increased the likelihood of an HIV test. Furthermore, the study cannot fully explain the mechanism of the vasculopathy found nor, in the absence of HIV viral particles or antigen in the vessel wall on histology, can it confirm that HIV directly caused the vasculopathy.

Questions remain regarding HIV assoappearances are quite different in extracranial large and intracranial medium vessels. Is this a spectrum of the same disease process affecting arteries with differing structural and physiological properties, or do the changes reflect vessel specific immunological and pathophysiological responses to HIV infection? The relatively immunocompetent state of the patients with large artery vasculopathy compared with the relatively immunodeficient state of those with medium intracranial artery vasculopathy in this study may support distinct mechanisms.

More important to clinicians working in low resourced settings is the implication of HIV vasculopathy for the management of the HIV infected stroke patient. Should some form of angiography be routine to search for intracranial vasculopathy? In ciated vasculopathy. The pathological high resourced settings possibly yes, but in low resourced settings only if the investigation is likely to change the patient's management, perhaps by limiting the search for conventional causes of stroke. It is not clear whether antiretroviral therapy (ARV) reverses HIV associated vasculopathy. ${ }^{3}$ Indeed, it remains to be seen whether ARV therapy, which alters the lipid profile, will increase atherothrombotic causes of stroke over decades of therapy. ${ }^{4}$ To add to the management dilemma, the role of secondary prevention of stroke with statins, blood pressure lowering agents and antiplatelet agents in HIV infected stroke patients without conventional stroke risk factors is uncertain. Given the epidemic of HIV infection and the increasing burden of stroke in Africa, we need large, well designed, prospective, community based case control studies, if possible with post-mortem examination, to clarify the impact of HIV and HIV therapy on the nature of stroke and stroke management. ${ }^{5}$ We also need further investigation of the nature of HIV vasculopathy, and to guard against a blind assumption that HIV infected stroke patients must have a vasculopathy if there is no other obvious cause for their stroke.

J Neurol Neurosurg Psychiatry 2007;78:1291. doi: 10.1136/jnnp.2007.122416

Correspondence to: Dr Myles Connor, Queen Margaret Hospital, Whitefield Rd, Dunfermline, UK; mconnor@staffmail.ed.ac.uk

Competing interests: None.

\section{REFERENCES}

1 Tipping B, de Villiers L, Wainwright $\mathrm{H}$, et al. Stroke in patients with human immunodeficiency virus infection. J Neurol Neurosurg Psychiatry 2007;78: 1320-24

2 Tipping B, de Villiers L, Candy S, et al. Stroke caused by human immunodeficiency virusassociated intracranial large-vessel aneurysmal vasculopathy. Arch Neurol 2006;63:1640-2.

3 Ranchod K, Modi M, Goolab V, et al. Human immunodeficiency virus associated intracranial aneurysms: Report of three adult patients with an overview of the literature. J Neurol Neurosurg Psychiatry 2007 (in press).

4 de Saint ML, Vandhuick O, Guillo P, et al. Premature atherosclerosis in HIV positive patients and cumulated time of exposure to antiretroviral therapy (SHIVA study). Atherosclerosis 2006; 185:361-7.

5 Connor MD, Walker R, Modi G, et al. Burden of stroke in black populations in sub-Saharan Africa. Lancet Neurology 2007;6:269-78. 